\title{
Oral Health in Women During Preconception and Pregnancy: Implications for Birth Outcomes and Infant Oral Health
}

\author{
Kim A. Boggess • Burton L. Edelstein
}

Published online: 1 July 2006

(C) Springer Science+Business Media, Inc. 2006

\begin{abstract}
The mouth is an obvious portal of entry to the body, and oral health reflects and influences general health and well being. Maternal oral health has significant implications for birth outcomes and infant oral health. Maternal periodontal disease, that is, a chronic infection of the gingiva and supporting tooth structures, has been associated with preterm birth, development of preeclampsia, and delivery of a smallfor-gestational age infant. Maternal oral flora is transmitted to the newborn infant, and increased cariogenic flora in the mother predisposes the infant to the development of caries. It is intriguing to consider preconception, pregnancy, or intrapartum treatment of oral health conditions as a mechanism to improve women's oral and general health, pregnancy outcomes, and their children's dental health. However, given the relationship between oral health and general health, oral health care should be a goal in its own right for all individuals. Regardless of the potential for improved oral health to improve pregnancy outcomes, public policies that support comprehensive dental services for vulnerable women of childbearing age should be expanded so that their own oral and general health is safeguarded and their children's risk of caries is reduced. Oral health promotion should include education of women and their health care providers ways to
\end{abstract}

K. A. Boggess $(\square)$

Department of Obstetrics and Gynecology, University of North Carolina at Chapel Hill School of Medicine,

CB 7516, Chapel Hill, NC 27599-7516

e-mail: kboggess@med.unc.edu

\section{B. L. Edelstein}

Department of Health Policy and Management Columbia, University Mailman School of Public Health,

New York, NY prevent oral disease from occurring, and referral for dental services when disease is present.

Keywords Oral health $\cdot$ Pregnancy $\cdot$ Infant caries

\section{Introduction}

A woman's preconception and pregnancy experience with the two most prevalent diseases of the mouth-periodontal disease and dental caries-not only influences her own oral health status but also may increase her risk of other diseases such as atherosclerosis [1-4], rheumatoid arthritis [5], and diabetes [6], impact pregnancy outcome [7-9], and her offspring's risk of developing early and severe dental caries [1013]. Although largely preventable through evidence-based interventions, both periodontal disease and caries in women of childbearing age are highly prevalent, particularly among low-income women and members of racial and ethnic minority groups. In addition, both periodontal disease and caries are typically asymptomatic for long periods of time with only intermittent painful exacerbations. The combination of high prevalence, insufficient treatment rates, missed preventive opportunities, and intermittent symptoms led the US Surgeon General to publish a report in 2001 on oral health in America characterizing dental and oral disease as a "silent epidemic" [14]. Socioeconomic factors, lack of resources to pay for care, barriers to access to care, and lack of public understanding of the importance of oral health and effective self-care practices all represent underlying reasons cited for observed inadequacies in oral health.

An important oral condition affecting many pregnant women is periodontal disease. Periodontal disease is a destructive inflammatory condition of the gingiva and bone that supports teeth. It is most commonly associated with a 
gram-negative anaerobic infection of these structures. Fluid that bathes the tooth at the gingival margin, known as gingival crevicular fluid, often contains inflammatory mediators and oral pathogens associated with periodontal disease.

The mechanisms underlying this destructive process involve both direct tissue damage resulting from plaque bacterial products, and indirect damage through bacterial induction of the host inflammatory and immune responses [15]. Destructive periodontal disease affects up to $15 \%$ of the population of childbearing age, with a relatively high proportion of pregnant women demonstrating some degree of periodontal disease $[7,8,16]$. Advancing age, smoking, and diabetes are risk factors for the development of periodontal disease [17]. Whereas periodontal disease is a chronic, local oral infection, systemic inflammation may also occur [15].

The second oral disease important to women of childbearing age because of its maternal-child health associations is dental caries. Dental caries is the pathologic process by which teeth "decay" and develop "cavities." It occurs when acid is produced at the tooth surface by cariogenic bacteria in the dental plaque that metabolize dietary carbohydrates. Acquisition of these cariogenic bacteria, dietary practices that govern the caries process, use of fluorides that dampen the caries process, and utilization of dental care all link mothers' and children's experience with tooth decay through biological, behavioral, and social pathways.

\section{Is maternal oral health linked to pregnancy outcome?}

\section{Preterm birth}

Preterm birth, delivery at less than 37 weeks' gestation, occurs in approximately $12 \%$ of all births $[18,19]$. Prematurity is the leading cause of neonatal morbidity and mortality in non-anomalous infants [20]. There are numerous and heterogeneous factors associated with preterm birth, such as low maternal body mass index, maternal smoking, and maternal infections [21]. In 1996, Offenbacher and colleagues [7] first reported a potential association between maternal periodontal disease and delivery of a preterm/low birthweight infant. In a case-control study of 124 pregnant women, they observed that women who delivered at less than 37 weeks' gestation or an infant $<2500 \mathrm{~g}$ had significantly worse periodontal disease than control women. The adjusted odds ratio for delivery of a preterm, low birth weight infant was $\sim 7$; these data led the authors to conclude that periodontal disease may represent a previously unrecognized and clinically significant risk factor for delivery of a preterm low birth weight infant [7]. Extrapolation from these data suggested that $18 \%$ of the preterm, low birth weight infants born annually might be attributable to periodontal disease, and thus account for a significant proportion of the $\$ 5.5$ billion annual hospital costs associated with the care of preterm/low birthweight infants. In a subsequent case-control study, Dasanayake et al. studied 55 pairs of women. Logistic regression indicated that mothers with 'healthy gingiva' were at lower risk for low birth weight infants [22]. Women in both of these casecontrol studies were examined at the end of pregnancy or after delivery, which does not convincingly prove an antecedent exposure and thus causality. Despite this limitation, these early studies led to the hypothesis that periodontopathic bacteria, primarily Gram-negative anaerobes, may serve as a source for endotoxin and lipopolysaccharides, which then increases local inflammatory mediators including $\mathrm{PGE}_{2}$, and cytokines, and that this increases systemic inflammatory mediators that can then lead to preterm birth [23]. Additionally, Jeffcoat et al. [9] examined the relationship between maternal periodontal disease and spontaneous preterm birth among 1313 pregnant women, and found that moderate/severe maternal periodontal disease identified early in pregnancy was associated with an increased risk for spontaneous preterm birth, independent of other traditional risk factors [9].

Despite these compelling data, it is important to recognize that other studies have failed to demonstrate any association between maternal periodontal disease and preterm birth. In a case control study conducted in London, Davenport et al. [24] examined 236 infants born at $<37$ weeks' gestation or $<2500 \mathrm{~g}$ and compared them to a random sample of 507 control infants born at $\geq 38$ weeks' gestation and weighing $\geq 2500 \mathrm{~g}$. The authors found no evidence for an association between delivery of a preterm, low birth weight infant and periodontal disease and somewhat surprisingly, found that deeper mean tooth pocket depths at delivery was associated with a reduction in the risk of delivery of a preterm, low birth weight infant [24]. The authors surmised that these discrepant findings might be due at least in part to racial differences in study populations. In a follow-up longitudinal study of 3738 women, Moore et al. [25] found no association between maternal periodontal disease and preterm birth. However, there was an increase in second trimester fetal loss rates among women with periodontal disease [25].

In an effort to better understand the possible mechanism behind the association between periodontal disease and preterm delivery, Offenbacher and colleagues [23] measured gingival crevicular levels of $\mathrm{PGE}_{2}$ and IL- $1 \beta$ in 48 mothers who delivered preterm, low birth weight infants compared to control women and discovered that gingival crevicular fluid levels of $\mathrm{PGE}_{2}$ were significantly higher in case compared to control women. Furthermore, among the primiparous women delivering preterm, low birth weight infants, a significant inverse association was demonstrated between birthweight and gestational age and gingival crevicular $\mathrm{PGE}_{2}$ levels [23].

It is not yet clear whether the relationship between periodontal disease and adverse pregnancy outcomes is causal 
or is a surrogate for another maternal factor. As further evidence to support the concept that maternal oral health is important for normal pregnancy outcome, other investigators have examined the effect of antepartum treatment of periodontal disease on preterm birth risk. Three published studies of antepartum versus delayed (postpartum) treatment of maternal periodontal disease demonstrate promise for this intervention for preterm birth prevention. The effect of periodontal interventions on pregnancy outcome was assessed in a prospective study designed to examine the relationship between periodontal disease and preterm low birthweight infants in a cohort of young, minority, pregnant and postpartum women. Of 164 women for whom birth outcome data were available, 74 were subjected to oral prophylaxis during pregnancy, and 90 received no periodontal treatment. The preterm/low birthweight rate was lower among women who received periodontal treatment compared to those who did not (13.5\% vs. $18.9 \%)$ [26]. Lopez et al. conducted a randomized clinical trial to assess the impact of periodontal treatment initiated during pregnancy versus delayed until postpartum on preterm low birthweight infant rates. The incidence of preterm/low birthweight infants in the antepartum treatment group was 1.8\% (3/163) and in the delayed/postpartum group was $10.1 \%$ (19/188), (odds ratio [OR] 5.5, 95\% confidence interval $[\mathrm{CI}] 1.7-18.2, P=0.001)$. Multivariable logistic regression analysis showed that periodontal disease was the strongest factor related to delivery of a preterm/low birthweight infant (OR 4.7, 95\% CI 1.3-17.1) [27]. The data from these two studies suggest that treatment of periodontal disease during pregnancy could reduce preterm/low birthweight infant rates [26, 27].

In a pilot intervention trial designed to assess the feasibility of conducting a trial to determine whether treatment of periodontal disease reduces the risk of spontaneous preterm birth, Jeffcoat et al. found that among women at high risk for preterm birth and presence of periodontal disease, scaling and root planning therapy initiated during pregnancy is tolerated by pregnant women and may reduce spontaneous preterm birth [28].

\section{Other adverse pregnancy outcomes}

Preeclampsia is a hypertensive disorder of pregnancy responsible for significant maternal and fetal morbidity and mortality. Preeclampsia affects up to 5\% of pregnant women [29]. The etiology of preeclampsia remains elusive. The underlying pathology may be related to a generalized intravascular hyperinflammatory state [29]. Some investigators have hypothesized a potential role for maternal periodontal disease as a risk factor for preeclampsia. In a retrospective analysis of data collected as part of the Oral Conditions and Pregnancy Study, Boggess et al. reported that women were at higher risk for preeclampsia if they had severe periodontal disease at delivery (adjusted odds ratio 2.4, 95\% confidence interval $1.1,5.3$ ), or if they had periodontal disease progression during pregnancy (adjusted odds ratio 2.1, 95\% confidence interval 1.0, 4.4) [30]. In a case-control study, Canakci et al. found that pre-eclamptic patients were 3.5 (95\% CI $=1.1-11.9)$ times more likely to have periodontal disease than normotensive patients $[P<0.01)$ [31]. In a study of 30 pregnant women, significantly higher periodontal probing depth and clinical attachment level scores were found among preeclamptic women compared with non-preeclamptic women. Gingival crevicular fluid levels of $\mathrm{PGE}_{2}, \mathrm{TNF}-\alpha$, and IL- $1 \beta$ levels were all significantly higher in the preeclamptic group [32]. Further study on the maternal and fetal inflammatory responses to chronic oral infection and on placental pathology in women with periodontal disease is needed to determine whether the relationship between periodontal disease and preeclampsia is causal or simply associative. If the relationship between maternal periodontal disease and preeclampsia risk proves causal in nature, then prevention of periodontal disease before pregnancy or treatment of periodontal disease during pregnancy may represent a novel approachs to the prevention of preeclampsia.

\section{Is maternal oral health linked to children's experience with tooth decay?}

Cariogenic bacteria are typically acquired by young children through direct salivary transmission from their mothers [10]. Factors influencing transmission are the levels of these bacteria in maternal salivary reservoirs, frequency and efficiency of transmission, and the child's receptivity to implantation, which is largely diet dependent. Additional factors include timing of transmission, which is affected by the window of infectivity and the age of the child, and the composition and flow of the child's saliva. The earlier the transmission and the more caries-supportive the diet, the earlier and more substantial the transfer will be. For this reason, mothers who have themselves experienced extensive tooth decay and therefore most likely harbor high titers of mutans streptococci in their saliva will more effectively transmit this infection vertically, thereby putting their young children at elevated risk for early childhood caries. Although maternal cariogenic bacteria can be isolated in the pre-dentate infant's mouth [11], these organisms become established in the dental plaque on the tooth surface only after teeth first appear at around six months of age. Because oral flora tends to remain stable over time, a woman's cariogenic flora before and during pregnancy anticipates her flora during the child's first years of life as well as the likelihood of transmitting infection early to her offspring. The lag time between infection and expression of a discernable cavity in a tooth depends upon additional factors, including the frequency of simple carbohydrate exposure in 
a child's diet, oral hygiene, and exposure to fluorides. The evidence that caries is frequently established as a pathologic process in the mouths of very young children is strong, as $28 \%$ of US children, over 4 million toddlers and preschoolers, experience one or more frank cavities by ages $2-5$ years [33].

Given the biological and behavioral pathways that govern intergenerational transmission of caries activity, disease management, and use of dental care, it is not surprising that disparities in dental caries among adults are mimicked among their children. As with adults, children of color and children of low-income families experience substantially more extensive and severe disease and less treatment than their peers without these risk factors [33]. Fortunately, despite the high prevalence of caries in women and children, this disease is readily preventable or manageable though early and regular dental care, exposure to fluoridated water, use of appropriate topical fluorides including those in toothpastes, application of sealants to primary teeth, and adoption of a health-promoting diet like that suggested in the Dietary Guidelines for Americans [34].

\section{Is preconception preventive oral health care the answer?}

It is intriguing to consider preconception, pregnancy, or intrapartum treatment of oral health conditions as a mechanism to improve women's oral and general health, pregnancy outcomes, and their children's dental health. Evidence is currently weakest for interventions that seek to reduce the incidence of preterm low birth weight through oral care. The mechanism of periodontal disease-associated adverse pregnancy outcomes is as yet unclear, and although it is hypothesized that if the 'insult' occurs early (either at conception or implantation) the risk is greater, no direct evidence to confirms that this is the case. However, given the strong relationship between oral health conditions and periodontal disease and general health and well-being, oral health care should be a goal in its own right for all individuals. If treatment of periodontal disease is going to impact pregnancy outcomes, then it is likely that the therapy will be of greatest benefit before or in very early pregnancy.

The science supporting interventions before, during, and after pregnancy to reduce caries transmission is much stronger. Educational and behavioral interventions that reduce caries activity through appropriate use of fluorides, dietary guidelines, chlorhexidine gels and varnishes, and xylitol [35], can reduce a woman's caries activity and salivary cariogenic flora, thereby improving her own oral health and, at the same time, also reducing the risk of transmission to her offspring. In two landmark Swedish studies [12, 13], children of mothers who had their cariogenic oral flora sup- pressed were less likely to experience cavities, more likely to develop cavities later if they were affected, and had fewer cavities than children of control mothers. Pregnancy is itself often regarded as an opportune time for anticipatory guidance and oral health education, and is a suitable time, particularly during the second trimester, for dental repair.

Access to oral health care during pregnancy

The CDC's Pregnancy Risk Assessment Monitoring System (PRAMS) reported that only $23-43 \%$ of pregnant women received dental care during their pregnancies [36, 37]-a rate only half to two-thirds of US women's overall use of dental services (67\%) (14). The PRAMS data revealed that overall, pregnant women covered by Medicaid were $24 \%-53 \%$ less likely to obtain a dental visit during pregnancy than women who are privately insured. Similarly, women who initiated prenatal care later than the first trimester, who did not intend the pregnancy, and who were poor were also less likely to obtain dental care [36]. In contrast, the Behavioral Risk Factor Surveillance System (BRFSS) revealed that $70 \%$ of pregnant women in the years 1999 and 2002 had received a dental visit in the prior 12 months [38]. One possible explanation for the higher level reported by BRFSS is that it includes three or more months of pre-pregnancy time, during which dental care utilization would be expected to reflect the national norm for women. However, in contrast to recognized disparities in dental care utilization [33], race and ethnicity were not significantly associated with dental care during pregnancy in the BRFSS study. The authors suggest that, "the prevalence of dental visits ... probably reflects factors such as prevailing attitudes toward dental care, provider availability and practice norms, and salient features of medical and dental care delivery within the state." Overall, women covered by Medicaid were $24 \%-53 \%$ less likely to obtain a dental visit during pregnancy than women who are privately insured. Similarly, women who initiated prenatal care later than the first trimester, who did not intend the pregnancy, and who were poor were also less likely to obtain care.

An important additional consideration is that dentists are reportedly reluctant to provide care to pregnant women because of concern about possible risks [39]. Current practice typically limits non-urgent dental treatment of pregnant women to the second trimester, as there is concern about possible teratogenic consequences during the first trimester and about the woman's comfort in the dental chair during the third trimester. A single study relating antepartum dental radiography with full term low birth weight raised concern about the safety of dental care during pregnancy [40], but was criticized for its methodology. Neither professional associations nor government agencies have promulgated any authoritative guidance regarding dental care of pregnant women, although multi-center NIH clinical trials are underway that will 
determine the impact of dental care for periodontal disease during pregnancy on preterm low birth weight outcomes. Currently, the New York State Department of Health is developing "Guidelines for Prenatal Care, Oral Health, and Child Health Professionals" that promotes routine use of dental care during pregnancy.

Independent of pregnancy, the presence and source of dental insurance coverage is an important predictor of dental care utilization, with publicly insured adults experiencing higher levels of oral diseases but less access to dental care. Medicaid is particularly significant to dental care of pregnant women as this program covers approximately $1 / 3$ rd of births in the US. However, states vary widely in adult Medicaid dental coverage, and at present only 7 jurisdictions providing comprehensive care to eligible adults. In contrast, low income pregnant women seeking dental services find themselves with no coverage in 8 states, coverage for only relief of pain or infection in 18 states or eligible for a limited range of services in 18 states. Three states (UT, LA, CA) have recently expanded dental benefits specifically to pregnant women in anticipation of reduced rates of unfavorable pregnancy outcomes. Pregnancy may be the only time that some low-income woman can readily obtain dental care as some state Medicaid programs provide adult dental coverage only to pregnant women or enhanced coverage during pregnancy.

\section{Conclusions and future directions}

Data are emerging to support a role for maternal periodontal disease as an infectious risk factor for preterm birth and other adverse outcomes of pregnancy. The prevalence of periodontal disease and the possibility of preterm birth prevention by treatment of oral infection make this a novel approach to improve the health and well being of our mothers and their soonto-be born children. Further studies to better understand the mechanism of periodontal disease-associated preterm birth will enable us to tailor treatment to those women who might benefit the most.

Data on the relationship between maternal and child experience with dental caries is well established. Therefore, regardless of the potential for improved oral health to improve pregnancy outcomes, public policies that support comprehensive dental services for vulnerable women of childbearing age should be expanded, so not only their own oral and general health is safeguarded but also so that their children's risk of caries is reduced. Particularly if NIH trials confirm that treating pregnant women for periodontal disease reduces the incidence of unfavorable birth outcomes, the Centers for Medicare and Medicaid Services should build on its September 2004 coverage expansions for pregnant women by stimulating the states to similarly expand oral health services for pregnant women.
The power of prevention needs to be brought to bear, as both periodontal disease and caries are overwhelmingly preventable through well recognized strategies including regular and effective home care for periodontal disease and use of fluorides and sealants for caries. To the degree that pregnancy provides a "teachable moment" in self-care and future childcare, prenatal education should universally adopt an oral health component. This educational intervention should prioritize those mothers who have suffered significantly from dental caries so that they can learn to effectively prevent transfer of this disease to their children.

To be effective, oral health promotion must first seek to educate women and their health care providers about the importance of oral health and must promote an understanding of their ability to prevent and manage both periodontal disease and caries and to thereby limit the personal and intergenerational consequences of both conditions.

\section{References}

1. Scannapieco FA. Position paper of the American Academy of Periodontology: periodontal disease as a potential risk factor for systemic diseases. J Periodontol. 1998;69:841-50.

2. Okuda K, Ebihara Y. Relationships between chronic oral infectious diseases and systemic diseases. Bull Tokyo Dent Coll. 1998;39:165-74.

3. Beck JD, Pankow J, Tyroler HA, Offenbacher S. Dental infections and atherosclerosis. Am Heart J. 1999;138:528-33.

4. Slade GD, Ghezzi EM, Heiss G, Beck JD, Riche E, Offenbacher S. Relationship between periodontal disease and C-reactive protein among adults in the Atherosclerosis Risk in Communities study. Arch Intern Med. 2003;163:1172-9.

5. Mercado F, Marshall RI, Klestov AC, Bartold PM. Is there a relationship between rheumatoid arthritis and periodontal disease? J Clin Periodontol. 2000;27:267-72.

6. Thorstensson H, Kuylenstierna J, Hugoson A. Medical status and complications in relation to periodontal disease experience in insulin-dependent diabetics. J Clin Periodontol. 1996;23:194 202.

7. Offenbacher S, Katz V, Fertik G, Collins J, Boyd D, Maynor G, et al. Periodontal infection as a possible risk factor for preterm low birth weight. J Periodontol. 1996;67:1103-13.

8. Moore S, Ide M, Wilson RF, Coward PY, Borkowska E, Baylis R, et al. Periodontal health of London women during early pregnancy. Br Dent J. 2001;191:570-3.

9. Jeffcoat MK, Geurs NC, Reddy MS, Cliver SP, Goldenerg RL, Hauth JC. Periodontal infection and preterm birth: results of a prospective study. J Am Dent Assoc. 2001;132:875-80.

10. Caufield PW, Cutter GR, Dasanayake AP. Initial acquisition of mutans streptococci by infants: evidence for a discrete window of infectivity. J Dent Res. 1993;72:37-45.

11. Berkowitz RJ. Acquisition and transmission of mutans streptococci. J Calif Dent Assoc. 2003;31:135-8.

12. Kohler B, Andreen I, Jonsson B. The effect of caries-preventive measures in mothers on dental caries and the oral presence of the bacteria Streptococcus mutans and lactobacilli in their children. Arch Oral Biol. 1984;29:879-83.

13. Kohler B, Bratthall D, Krasse B. Preventive measures in mothers influence the establishment of the bacterium Streptococcus mutans in their infants. Arch Oral Biol. 1983;28:225-31. 
14. Oral Health in America: A Report of the Surgeon General. In: Services USDoHaH, ed. U.S. Department of Health and Human Services, National Institute of Dental and Craniofacial Research, National Institutes of Health. Rockville, MD: U.S. Department of Health and Human Services, 2000.

15. Genco RJ. Host responses in periodontal diseases: current concepts. J Periodontol. 1992;63:338-55.

16. Davenport ES, Williams CE, Sterne JA, Sivapathasundram V, Fearne JM, Curtis MA. The east London study of maternal chronic periodontal disease and preterm low birth weight infants: study design and prevalence data. Ann Periodontol. 1998;3:213-21.

17. Genco R. Risk factors for periodontal disease. In: Rose L, Genco R, Mealey B, Cohen D, editors. Canada: BC Decker, 2000.

18. Martin J, Hamilton B, Ventura S, Menacker S, Park M. Births: Final data for 2000. Mon Natl Vital Stat Rep. 2002;50:1-104.

19. Martin JA, Hamilton BE, Sutton PD, Ventura SJ, Menacker F, Munson ML. Births: final data for 2002. Natl Vital Stat Rep. 2003;52:1-113.

20. Mathews T, Menacker S, MacDorman M. Infant mortality statistics from the 2001 period linked infant brith/death data set. Natl Vital Stat Rep. 2003;52:1-27.

21. Kramer MS. The epidemiology of adverse pregnancy outcomes: an overview. J Nutr. 2003;133:1592S-6S.

22. Dasanayake AP. Poor periodontal health of the pregnant woman as a risk factor for low birth weight. Ann Periodontol. 1998;3:20612.

23. Offenbacher S, Jared HL, O'Reilly PG, Wells SR, Salvi GE, Lawrence HP, et al. Potential pathogenic mechanisms of periodontitis associated pregnancy complications. Ann Periodontol. 1998;3:233-50.

24. Davenport ES, Williams CE, Sterne JA, Murad S, Sivapathasundram V, Curtis MA. Maternal periodontal disease and preterm low birthweight: case-control study. J Dent Res. 2002;81:313-8.

25. Moore S, Ide M, Coward PY, Randhawa M, Borkowska E, Baylis R, et al. A prospective study to investigate the relationship between periodontal disease and adverse pregnancy outcome. Br Dent J. 2004;197:251-8; discussion 247.

26. Mitchell-Lewis D, Engebretson SP, Chen J, Lamster IB, Papapanou PN. Periodontal infections and pre-term birth: early findings from a cohort of young minority women in New York. Eur J Oral Sci. 2001;109:34-9.

27. Lopez NJ, Smith PC, Gutierrez J. Periodontal therapy may reduce the risk of preterm low birth weight in women with periodontal disease: a randomized controlled trial. J Periodontol. 2002;73:911-24.
28. Jeffcoat MK, Hauth JC, Geurs NC, Reddy MS, Cliver SP, Hodgkins $\mathrm{PM}$, et al. Periodontal disease and preterm birth: results of a pilot intervention study. J Periodontol. 2003;74:1214-8.

29. Sibai BM, Gordon T, Thom E, Caritis SN, Klebanoff M, McNellis $\mathrm{D}$, et al. Risk factors for preeclampsia in healthy nulliparous women: a prospective multicenter study. The National Institute of Child Health and Human Development Network of Maternal-Fetal Medicine Units. Am J Obstet Gynecol. 1995;172:642-8.

30. Boggess KA, Lieff S, Murtha AP, Moss K, Beck J, Offenbacher S. Maternal periodontal disease is associated with an increased risk for preeclampsia. Obstet Gynecol. 2003;101:227-31.

31. Canakci V, Canakci CF, Canakci H, Canakci E, Cicek Y, Ingec M, et al. Periodontal disease as a risk factor for pre-eclampsia: a case control study. Aust N Z J Obstet Gynaecol. 2004;44:568-73.

32. Oettinger-Barak O, Barak S, Ohel G, Oettinger M, Kreutzer H, Peled M, et al. Severe pregnancy complication (preeclampsia) is associated with greater periodontal destruction. J Periodontol. 2005;76:134-7.

33. Beltran-Aguilar ED, Barker LK, Canto MT, Dye BA, Gooch BF, Griffin SO, et al. Surveillance for dental caries, dental sealants, tooth retention, edentulism, and enamel fluorosis-United States, 1988-1994 and 1999-2002. MMWR Surveill Summ. 2005;54:143.

34. Fitzsimons D, Dwyer JT, Palmer C, Boyd LD. Nutrition and oral health guidelines for pregnant women, infants, and children. J Am Diet Assoc. 1998;98:182-6, 189; quiz 187-8.

35. Gussy MG, Waters EG, Walsh O, Kilpatrick NM. Early childhood caries: current evidence for aetiology and prevention. J Paediatr Child Health. 2006;42:37-43.

36. Gaffield ML, Gilbert BJ, Malvitz DM, Romaguera R. Oral health during pregnancy: an analysis of information collected by the pregnancy risk assessment monitoring system. J Am Dent Assoc. 2001;132:1009-16.

37. Mangskau KA, Arrindell B. Pregnancy and oral health: utilization of the oral health care system by pregnant women in North Dakota. Northwest Dent. 1996;75:23-8.

38. Timothe P, Eke PI, Presson SM, Malvitz DM. Dental care use among pregnant women in the United States reported in 1999 and 2002. Prev Chronic Dis. 2005;2:A10.

39. Livingston HM, Dellinger TM, Holder R. Considerations in the management of the pregnant patient. Spec Care Dentist. 1998;18:183-8.

40. Hujoel PP, Bollen AM, Noonan CJ, del Aguila MA. Antepartum dental radiography and infant low birth weight. Jama. 2004;291:1987-93. 